\title{
Investigación y docencia en salud mental (II): ¿Queda algún futuro para la "Asistencia Centrada en el Consultante" y el pensamiento clínico?
}

RESUMEN: El trabajo parte de una reflexión epistemológica sobre la investigación en psicoterapia y una descripción de las áreas, métodos y condicionantes para el futuro de la misma. Se describen asimismo algunas consecuencias ilustrativas de los sesgos que han venido mostrándose anteriormente, tanto de la "perspectiva Bambi" como de la "perspectiva Godzilla". Se plantean como ejemplos ilustrativos los siguientes: la santificación del "managed care"; el efecto Utah en el lanzamiento de nuevos neurolépticos y antidepresivos; los resultados negativos de los metaanálisis de una técnica que sigue practicándose y defendiéndose, como es la TEC; los resultados positivos pero poco conocidos de algunas técnicas psicológicas elementales en el cuidado de los pacientes graves y sus familiares así como de la depresión puerperal; los resultados negativos $o$ inciertos de determinadas técnicas "contraculturales", etc. Para terminar, se realizan una serie de propuestas integradoras, tanto para la investigación como para la docencia en salud mental, orientadas por un intento de soslayar los dogmatismos y sectarismos habituales.

PALABRAS CLAVE: Investigación Formación Continuada - Docencia - Salud Mental - Psicopatología - Psiquiatría - Asistencia - Técnicas psicológicas Psicoterapia.
SUMMARY: Paper begins with an epistemological reflection on psychotherapy research and a description of areas, methods and condition ants for its future. Some shunts of Bambi's perspective and Godzilla's perspective are described. Some illustrative examples are considered: the sanctification of "managed care", the Utah effect on marketing of new neuroleptics and antidepressants, the meta-analysis negative results about ECT, the positive but almost unknown effects of some elementary psychological techniques on severe disorders care and on puerperal depression, the negative results of some "contra cultural" techniques... To end, a series of integrative proposals for research and teaching in mental health are developed --with the common objective to avoid the sectarian and dogmatic trends almost habitual in that field.

KEY WORDS: Research - Continuous Training - Teaching - Mental Health Clinical Thought - Psychopathology Psychiatry - Sanitary Care - Psychological Techniques - Psychotherapy. 
En la primera parte de este trabajo he intentado plantear algunas reflexiones sobre la conyuntura socio-económica de nuestras instituciones de asistencia, así como una tipificación de las orientaciones que se proponen en esa coyuntura para el desarrollo de la investigación y la docencia en el ámbito de la salud mental.

\section{Algunas reflexiones epistemológicas} sobre la investigación en (psico)terapias.

Nuevamente, en este apartado, no me basaré en autores de mi propia orientación y marco de referencia (Wallerstein, Luborsky, Horowitz, Trujillo por ejemplo entre los extranjeros; o Avila, Poch o Castillo entre los nacionales). Como ya hice en 1995(5) (7-10), intentaré desmarcarme de mis propios resabios dogmáticos basándome en autores de marco de referencia diferente al mío. De entrada, me fijaré en las propuestas de Kazdin(31-35).

Como intenté mostrar en la revisión arriba citada(10), hace ya más de cuarenta años que la literatura científica respecto a las psicoterapias se ha llenado de asertos acerca de una variable sobre la que hoy parece bastante irrelevante investigar. Es indudable que, a pesar de sus errores, falacias y deformaciones conscientemente mantenidas (4) (10), los trabajos de Eysenck, en vez de producir el objetivo fundamental buscado (demostrar la ineficacia de la psicoterapia) produjeron una enorme revitalización de todas las psicoterapias con "aspiraciones científicas" y, en general, de la investigación en psicoterapia.

Por otra parte, si se tienen en cuenta los datos proporcionados en la tabla
1, sobre los problemas de los tratamientos exclusivamente farmacológicos de los trastornos mentales más prevalentes, la investigación en psicoterapia adquiere un valor no sólo psicológico y psicopatológicio, sino en general, sanitario. Por eso, investigadores como Kazdin (32) (35) proponen que actualmente, en vez de investigar la eficacia de tales técnicas, hoy ya reconocidas como eficaces, se dedicaran más esfuerzos a las áreas resaltadas en la tabla 2.

En primer lugar, habría que concentrar la investigación en los estudios con muestras clínicas. Esa propuesta puede resultar sorprendente para quien no conozca de cerca la literatura universitaria anglosajona sobre "investigación en psicoterapia": pero es que resulta asombroso observar cuántos asertos y aseveraciones se han hecho al respecto a partir de investigaciones sobre "psicoterapias" realizadas con voluntarios, estudiantes, voluntarios pagados, análogos, etc. Hoy ya podemos decir que cualquier parecido entre gran parte de las "terapias" de dichos estudios y la Psicoterapia que realizamos en settings privados o públicos los psicoterapeutas no suele ser sino pura coincidencia. Creo que hay un estado de opinión creciente respecto a la escasa validez de ese tipo de investigaciones, hasta ahora tan frecuentes (entre otras razones, porque los sistemas de salud de los USA son muy poco solidarios y "equitativos": ¿de dónde sacar pues los voluntarios clínicos?).

Otra área que habría que priorizar sería la investigación de los efectos específicos sobre problemas, cuadros, 
personalidades o poblaciones con características también específicas: de poco valen asertos tales como "la psicoterapia de tipo " $x$ " es eficaz" o incluso, "es eficiente". No son fiables estudios que no delimiten mucho más la población, los problemas y los resultados: psicoterapias del tipo "X", aplicadas según principios, técnicas y distribución espacio-temporal definidos ( $\left.T, T^{\prime}, T^{\prime \prime} . ..\right)$, a una población de características C (C,C', C'”...), aquejada de los diagnósticos y problemas determinados (P, P',P'), proporcionan resultados determinados ( $R, R$ ', $R$ '...) en cuanto a eficacia, eficiencia, accesibilidad, seguridad, resultados a largo plazo, cambios en la calidad de la vida....

Otra área a priorizar es el área de comorbilidad, que dice Kazdin. Pero mi opinión es que aquí existe otro sesgo epistemológico e ideológico de base, ante el cual yo no hablo de comorbilidad y prefiero hablar de codiagnóstico. Hablar de comorbilidad, que es el término de moda, es dar por sentado ya que existen dos "entidades morbosas" que coinciden en la misma persona. Pero ¿qué y quién las ha definido como "entidades morbosas", y más con una clasificación "ateorética" como el DSM-IV'? Empíricamente no está demostrado que sean entidades morbosas esas que se hace coincidir o que coinciden en los estudios. Se trata tan sólo de "situaciones de codiagnóstico", en las cuales se definen los resultados de sendas operaciones formales. Pero como a los "ateoréticos" eso de las operaciones formales "no les va", tal vez por ello (y para darle más relevancia biológica al codiagnóstico) se suele dar el salto: un salto que, insisto, desde el punto de vista empírico y epistemológico es un salto en el vacío.

Ahora bien: investigar sobre los codiagnósticos y sobre las posibilidades de la psicoterapia y la combinación de psicoterapias y fármacos en los cuadros de codiagnóstico es un tema de gran relevancia. Como lo es la siguiente recomendación de Kazdin, proponiendo centrar la investigación en poblaciones infradotadas de medios psicosociales de tratamiento y poblaciones de riesgo: por ejemplo, minorías étnicas. Para los que trabajamos hace años en barrios con alta proporción de miembros de la etnia gitana, resultaría de gran interés el que equipos con más medios, formación y/o conocimientos investigaran sobre las técnicas y vías terapeúticas para este tipo de poblaciones... $\mathrm{O}$ para los inmigrantes del Sur, un problema creciente en todos los países y sociedades desarrolladas ... del hemisferio Norte. Todos ellos son grupos poblacionales que nos plantean cuadros y dificultades asistenciales muy severas que no solo señalan la necesidad de una investigación biológica con nuevas bases, sino también de investigaciones psicológicas y psicosociales e incluso sociologicas.

En este ámbito suele darse un dato clave que cada año pongo de relieve: en zonas geodemográficas $o$ áreas básicas en las cuales el centro de salud trabaja hasta con el 150 $20 \%$ de habitantes de etnia gitana $o$ de inmigrantes del sur, habitualmente ninguna autoridad administrativa, ni incluso los propios coordinadores médicos, ayudan a que el equipo asistencial posea un conocimiento 
directo de en qué consisten las costumbres gitanas, magrebies, guineanas o gambianas vinculadas con temas de salud (mental). Ello complica enormemente nuestra comprensión de las quejas y problemas de los caló, los norteafricanos o los centroafricanos por parte del personal asistencial payo. Me refieron por ejemplo a su idiosincrasia con respecto al duelo, la pérdida, la enfermedad, el cuidado de los hijos pequeños, el uso de la desnudez, exploratoria o no, etc.

Es el propio Kazdin quien propone, a continuación -aspecto en el que coincido plenamente- la investigación de las técnicas infraestudiadas pero frecuentemente utilizadas "como son las técnicas psicoanalíticas". Por poner un ejemplo, en Cataluña las dos terceras partes de los centros comunitarios de salud mental consideran de sí mismos que su modelo asistencial es el psicoanalítico --o el mixto que incluye el psicoanalítico--: por tanto, las técnicas terapéuticas que utilizan son las menos sujetas a investigaciones empíricas de todas las psicoterapias descritas.

En cuanto a la investigación de la combinación de tratamientos, la simplificación de tratamientos, los sistemas breves y las técnicas adaptadas, lo único que puedo decir aquí es que ha sido uno de mis intereses en los diez o quince últimos años, interés que se ha resumido en las obras colectivas publicadas por la Unitat de Salut Mental que dirijo (36) (37) y en diversos estudios empíricos citados en la bibliografía (38-43). Por último, del séptimo apartado de la tabla, que no es de Kazdin sino mío (la necesidad de investigar otras características de la calidad de las terapias además de la eficacia: Tabla 3 ), ya llevo dichas algunas palabras, tanto en este trabajo como en otros anteriores (6) (10) $(36-38)$.

Desde ese punto de vista (tabla 3 ), gran parte de las investigaciones de cualquier tipo, biológico, psicológico o social, se están centrando hoy en día en la eficacia, definida más o menos como la capacidad de una técnica para solucionar problemas, así como en la eficiencia, definida como el consumo o costos de una técnica. Una Joint Comission americana al respecto(44) ha delimitado mucho más ampliamente esas "características de la calidad de una terapia cualquiera", característias que he ampliado y sistematizado en la tabla 3: al menos pueden delimitarse once características diferentes a investigar en cada una de las técnicas. En algunos casos, la sóla discriminación de esas características arrumba muchas investigaciones anteriores, que se muestran ante ellas demasiado burdas o ideológicamente sesgadas. Por ejemplo: de poco vale una técnica muy eficaz, que se ha comprobado como muy eficaz, pero cuyos practicantes, tal vez por motivos intrínsecos a la técnica -por ejemplo, porque su práctica resulta a medio o corto plazo muy absorbente y reiterativa-, no pueden seguirla practicando al menos unos años: ¿no les suena a ustedes como uno de los resultados a medio plazo de más de una técnica conductual en la realidad pragmática de nuestros equipos?

A otro nivel, de nada vale una técnica muy eficaz e incluso muy eficiente si luego la situación media del país o de la 
comunidad no permite que sea aplicada a un nivel medio (efectividad). El ejemplo, proveniente de otro campo sanitario, se ha hecho tristemente célebre: al no considerar los problemas de efectividad, sanitarios que colaboraron con los intereses de las empresas trasnacionales de la lactancia artificial en su intento de exportarla al Africa subsahariana, colaboraron también de forma más o menos ignorante en un real genocidio, con el resultado de centenares de miles o incluso millones de recién nacidos muertos por infecciones $y$ afecciones digestivas del lactante. Porque el nivel medio de desarrollo tecnológico no daba para esas técnicas, suponiendo que fueran técnicas oportunas -y no tan sólo la consecuencia de un "reparto mundial de excedentes" y mercados de material "sanitario"-.

En el mismo sentido hablaría de la necesidad de realizar investigaciones acerca de la oportunidad y la accesibilidad: de poco vale hoy una técnica que se podrá aplicar tan sólo en un futuro remoto tras de grandes lista de espera. Es el caso de algunas técnicas sistémicas formalizadas, de muchas psicoterapias psicoanalíticas de niños formalizadas, de algunas psicoterapias de grupo cláásicas... (37) (41). Es posible que resulten más útiles las técnicas adaptadas, mixtas o menos formalizadas: por ejemplo, los "grupos de acogida y diagnóstico", las técnicas grupales (diferenciadas de la "psicoterapia de grupo"), las técnicas abreviadas o mixtas, etc. Son técnicas menos formalizadas, más "soft", posiblemente menos eficaces e incluso (aún) menos investigadas, pero que tal vez permitan aproximaciones más accesibles y oportunas al sufrimiento mental de la población... O al balance entre autonomía y heteronomía. O a la calidad de vida de los consultantes y sus allegados, tema sobre el cual ya existen escalas y no solo reflexiones y comunicaciones clínicas(45). En definitiva, si investigáramos esas otras características y tuviéramos en cuenta esos otros datos, creo que llegaríamos a una situación que podríamos definir como la de una investigación científica y tecnológica eficiente y adecuada (para la situación de nuestras redes asistenciales).

Pero ¿cuáles son, en general, las condiciones actuales para una investigación científica o tecnológica eficiente y adecuada, tal como son definidas por los teóricos de la investigación? Aquí me voy apoyar, nuevamente, en teóricos de otro marco de referencia, tales como Lynn y Garske, en su trabajo de 1988(46). (cfr. un resumen en la tabla 4). Desde esa perspectiva, lo primero que hemos de admitir, es que resulta básico en la investigación sobre terapias contar con grupos homogéneos de consultantes con problemas específicos e idénticos, es decir con los ECA (los estudios aleatorizados controlados). A pesar de los problemas mencionados páginas más atrás, y de nuestra experiencia anterior con estudios de este tipo irrelevantes, sesgados o falseados, hemos de admitir que, ciertamente, dentro de los estudios científicos son los más fiables en sus resultados. Pero ello nos autoriza a afirmar también -un poco provocativamente, es verdad- que, fuera de esos estudios ECA y de los estudios estrictamente experimentales, el resto de estudios poseen en principio el mismo nivel de valor teórico y heurístico que las comu- 
nicaciones de un clínico honesto y con suficiente experiencia (y existen investigaciones empiricas a favor de este aserto (46) (47). Todo estudio no aleatorizado controlado tiene una validez muy dudosa, sobre todo si además se está basando en reducionismos epistemológicos importantes.

Con esos datos en mano ¿cómo realizar estudios aleatorizados controlados en nuestra práctica habitual? Garske y Lynnn afirman que los grupos deben ser homogéneos, de consultantes con problemas específicos e idénticos, con muestras suficientemente numerosas (al menos, se siguen exigiendo entre 20 y 40 individuos, a pesar de la "estadística de las muestras pequeñas"), y que esos individuos deben estar asignados aleatoriamente a las condiciones experimentales (grupo experimental o grupo control).

En el campo de la investigación en técnicas de psicoterapia y, en general, en "psicoterapia y psicofármacos", esa "asignación aleatoria" hace irrumpir en nuestros (posibles) estudios múltiples problemas éticos y problemas de honestidad y ética científica.

Para ponerles un ejemplo sufcientemente conocido y estudiado de lo que les hablo, simplemente recordaré el aqui "experimento Tuskegee" o de "Evolución de la sifilis en macho negro" - asi se llamaba el trabajo--: en él, sin contar con los participantes, se sometio a decenas de hombres de color de los estados sureños norteamericanos durante 4 décadas a un estudio de la evolución natural de la síflis (sin ninguna técnica de cuidados médicos para los que cayeron en el grupo de control).

Otro ejemplo en el mismo sentido serian las experimentaciones en los campos de concentración nazis, que hace unos pocos años algunos científicos proponian recuperar para la investigación: es el caso de los estudios sobre hipotermia del famoso Dr. Rascher, un amigo personal de Himmler --al que al mismo Himmler ejecuto, por cierto. Pero cuando los investigadores han tenido acceso a los datos de los "experimentos" en los cuales 300 judios y rusos fueron sumergidos en agua fría durante horas para analizar la resistencia a la hipotermia, se encontraron con una realidad que ya la muerte post-prueba de todos los sujetos "experimentales" anunciaba: a pesar del supuesto rigor y la efectividad de la "ciencia alemana", los estudios estaban llenos de acientificismo y falta de rigor metodológico, resultando, por tanto, completamente inútiles para la ciencia (48). En el mismo sentido, ya Beecher habia publicado un informe en 1966 con multitud de ejemplos de investigación médica en los USA donde los derechos humanos más elementales fueron sistemáticamente conculcados(49). Habrá que cuidar en nuestras investigaciones en salud mental y psiquiatría no repetir ese tipo de licencias, ni que sea a "escala menor".

La siguiente condición para la validez de las investigaciones empíricas que Garske y Lynn definen consiste en que 
los terapéutas han de estar igualados en cuanto a variables críticas: ello implica que la formación, experiencia y actualización en la técnica objeto de estudio de los terapeutas han de ser cuidadosamente controladas en el estudio, una condición que, en mi experiencia, pocas veces se cumple. Como en numerosas ocasiones, en las investigaciones publicadas sobre los efectos de las psicoterapias, en especial en las investigaciones más ideológicamente sesgadas, no se cumple la condición de "grupos de control cuidadosamente diseñados". Diseñar un grupo de control para una investigación clínica es un asunto lleno de problemas éticos, metodológicos y téricos, que aquí no puedo sino apuntar. Ese diseño de los grupos de control ha de incluir aquéllos que son necesarios para rechazar las hipótesis alternativas, tales como los efectos placebo y los efectos de la atencion, las expectativas y el contacto personal. Esos grupos de control y, en general, toda la experiencia, han de tener cuenta el principio de "crisálida" (o de "idealización juvenil":en sus primeros momentos de existencia, casi todas las terapias dan unos resultados excelentes); o la realidad de que, en temas de salud mental, a causa de la importantes efectos de la relación asistencial, no es posible diseñar grupos de "no-tratamiento", y resulta sumamente difícil el diseño e incluso la definición de "grupos de tratamiento mínimo" o "estándar"...

Acerca de este tema les diría que en más de 30 años de trabajo en salud mental tanto a nivel clínico, como docente e investigador, solo una vez me han consultado investigadores de orientación diferente sobre la metodo- logía de su propia investigación. Y sin embargo, parece que, para reducir la importancia de los efectos placebo, del efecto Rosenthal, de los efectos de las expectativas y el contacto o relación personal por comparación a los aspectos especificos del tratamiento, parecería bastante lógico recurrir a un profesional de un marco de referencia diferente al de los investigadores y, más aún, a un profesioanl de orientación psicoanalítica. En el caso en el que sí fuí consultado se trataba de una investigación de orientación conductual sobre colon irritable y la consulta creo que fué sumamente adecuada: se me pidió que criticara desde el punto de vista psicoanalítico el planteamiento de la investigación conductual. Se trataba de un equipo coordinado por el Prof. R. Bayés. Pero ¿cuántas investigaciones, antes de llevarse a cabo, son presentadas en nuestro medio a los clínicos o a los teóricos de la orientación o marco de referencia opuesto para que critiquen previamente la metodología, objetivos, criterios...?

Desde luego que si los problemas muestrales son desbordantes o no nos vemos capaces de superarlos, siempre nos queda la posibilidad de utilizar diseños de caso único (o de $\mathrm{N}=1$ ). Pero hemos de reconocer que, por hoy, tales diseños e investigaciones parece que siguen siendo poco valorados por investigadores y clínicos, así como por las publicaciones "de prestigio internacional" $\mathrm{y}$ "bancos de datos computerizados".

Otra de las condiciones de Garske y Lynn para una investigación eficiente en 
este ámbito es el uso de medidas múltiples del cambio, medidas utilizadas desde varias perspectivas: que varíen en su contenido (por ejemplo, conductual, cognitivo y afectivo) y en su fuente (por ejemplo, paciente, ambiente, terapeuta y observador); que se administren en varias oportunidades en el estudio: por ejemplo, antes y después de un período de tratamiento, y en el seguimiento (es decir, "en un período de tiempo fijado después del final del tratamiento"). En este tema, creo que vale la pena recordar un principio en el que vengo insistiendo desde hace años (4) (6) (10), como otros autores hacen más recientemente(49): para conocer los resultados a nivel biopsicosocial de cualquier tratamiento en salud mental, el período de seguimiento mínimo ha de ser de dos años. Un período más realista y que da mayor validez al estudio sería de cinco años para arriba... Debido a los efectos placebo y Rosenthal, a los valores terapéuticos de la relación, y a diversos tipos de contaminación experimental, en nuestro ámbito de la salud (mental) la validez de cualquier estudio sobre cualquier terapia que no siga los casos al menos durante esos cinco años es sumamente discutible. Aunque sea un estudio aleatorizado controlado, desde luego.

La siguiente condición de Garske y Lynn es que la muestra y los procedimientos deben estar bien descritos en el estudio. Pero, con poco complejo que sea dicho estudio, aparece un "pequeño problema": si ustedes describen bien la muestra y los procedimientos, ninguna revista que no sea la de un amigo les va a publicar ese estudio. Entre otros motivos, porque el original se habrá hecho demasiado largo: desde luego, no esperen publicarlo en ninguna de las revistas internacionales con más prestigio y penetración si no son conocidos del Consejo de Redacción. Y así pasa luego lo que pasa: el "fenómeno Darsee".

A esas condiciones creo que hay que añadirle, en el caso de la investigación en psicoterapia, la obligación de que la supuesta técnica aplicada sea estudiada ante los "tribunales de estilo" adecuados, que autentifiquen su fiabilidad y su soporte teórico-técnico: demasiado a menudo se investigan técnicas terapéuticas cuyo parecido con las habitualmente aplicadas en los medios clínicos es pura coincidencia. Por ejemplo, la técnica psicoanalítica aplicada en algunas de las investigaciones computerizadas y/o poligráficas sobre los acontecimientos de una sesión psicoanalítica o de psicoterapia psicoanalítica es muy dudoso que siga poseyendo los elementos básicos de encuadre interno y encuadre externo de un tratamiento psicoanalíitico o psicoterapéutico típico (cfr., por ejemplo, 50). Sería recomendable, por ello, que las investigaciones fueran consultadas en cuanto a la técnica terapéutica utilizada a expertos sobre el tema y/o a las asociaciones de terapeutas.

A todas estas condiciones, creo que habría que añadirle una serie de condicionantes reales provenientes de la práctica, la pragmática de la investigación científica en nuestros días, del "¿Para qué se publica?" Lejos de la ilusión de que en ciencia se publica "por aumentar el conocimiento científico o tecnológico", hay suficientes datos como para pensar 
que, hoy por hoy, la pragmática de la publicación científica está presidida por la búsqueda del beneficio personal y la carrera profesional de los investigadores. Ello supone, como hemos visto desde diferentes perspectivas, un enorme lastre en las publicaciones y bancos de datos científicos, y favorece más que ocasionales manifestaciones del "fenómeno Darsee". Por otro lado, hoy está más claro que nunca, al menos en los países y sectores científicos secundarios del Imperio, que es imprescindible pertenecer a una "escuela" o "colegio (más o menos) visible" para que cualquier intento de publicación sea valorado, así como para difundir las investigaciones (a menos que se use de forma descarada y organizada el "efecto Utah"). Otro condicionante no menor de la pragmática de la investigación en nuestra coyuntura y formaciones sociales proviene del hecho de que para financiar y difundir la investigación, en los centros y sectores secundarios, hasta el $85 \%$ del tiempo del equipo ha de dedicarse a rellenar formularios, peticiones y a labores de "relaciones públicas"... Desde luego, si no se pertenece a una institución, como la universidad o algunos hospitales, que poseen un departamento encargado de hacerlo. Es un "efecto secundario" de los controles para justificar la provisión de fondos para investigación, siempre escasos para la demanda, y un "efector primario" de la creciente burocratización de la Universidad, los Hospitales y las instituciones científicas en nuestros países y sectores secundarios de la investigación.

El resultado de todos estos condicionantes pragmáticos que he citado últimamente y de algunos otros más estructurales citados más arriba es que, en el mundo científico y docente actual, en último extremo lo que recibimos no lo aceptamos por científico (es decir, como conocimientos comunicables, verificables, apofánticos y no-contradictorios), sino por confianza ( $₫ \circ \mathrm{fe}$ ?). Confianza (o fe) en los amigos, conocidos, miembros del "colegio invisible" o simpatizantes que escriben los trabajos o las pre-publicaciones. Y eso puede llevarnos por ejemplo a aceptar durante decenios como las "conductas sexuales infantiles comunes" y como datos sobre la homosexualidad masculina, los descritos en Kinsey (51) (52). Pero hay investigaciones actuales que afirman que tales "datos" en buena parte procedían ... de un pedófilo acusado de abusar de 317 adolescentes, realidad que, oculta bajo el "efecto Utah", no ha llegado a nosotros hasta cincuenta años después (53) (54).

\section{Algunas consecuencias ilustrativas.}

En este último apartado voy a intentarles ilustrar las últimas afirmaciones generalizadoras realizadas, así como algunas de las iniciales, con una serie de ejemplos actuales que tienen que ver con nuestra pragmática y nuestras técnicas asistenciales. En varias de estas ilustraciones utilizaré datos procedentes de las revisiones meta-analíticas de la Fundación Cochrane, sin que ello signifique, desde luego, hipostasiar el valor de una organización de la búsqueda de evidencias científicas (la Medicina basada en la Evidencia o "en Pruebas") que seguirá siendo útil, no tanto por el método en sí, sino mientras conserve una cierta imparcialidad y, sobre todo, una cierta independencia con los poderes fácticos. Insisto en que mi uso de los datos propor- 
cionados por dichos metaanálisis tiene que ver, sobre todo, con el valor que le concedo a los estudios y a la trasparencia de las revisiones efectuadas, pero no tanto con un "apuntarse acrítico" a una nueva "moda metodológica" que promete la solución de los problemas citados anteriormente de la investigación y la publicación científica. Entre otras razones porque, probablmente, esos problemas son irresolubles en sí mismos, pues tienen que ver, como he intentando ilustrar en este trabajo, con la interpenetración entre la Ciencia, la Tecnología y el Poder (cfr. un resumen de las ventajas y problemas de la MBE por ejemplo en (56) (59).

La primera ilustración que les quería mencionar se halla de gran actualidad en varias comunidades del país: al parecer "desencantados" de las parcialidades e insuficiencias en el "manejo de casos" de los clínicos, y de los derroches, retrasos, parcialidades e insuficiencias de los sistemas habituales de atención clínica de los pacientes con "trastornos mentales severos" (TMS) por parte de aquéllos, algunos gestores del "primer mundo" han propuesto el sistema del "Managed Care" o los más modernos "Tratamientos Comunitarios Asertivos" (ACT): como cuesta lograr que clínicos a los que se ha desprogramado y desmotivado para la atención globalizadora y el cuidado de los pacientes según el sistema de "clínico referente" apoyado adecuadamente por grupos de reflexión y/o supervisión (36) (37), se inventa el sistema del CM o los ACT: el gran descubrimiento es que a cada paciente se le asigna un "case manager" que determina las necesidades personales, desarrolla un plan de cuidados, procura que sean proporcionados esos cuidados, monitoriza la calidad de los mismos y mantiene el contacto con el paciente(60). Es decir, que realiza el trabajo del "clínico referente" que nosotros proponíamos desde 1981 y 1992(61) (36)... pero, al parecer, sin clínica: los clínicos (psiquiatras, psicólogos, trabajadores sociales, enfermeros...) se convierten así en "operarios de una cadena productiva de cuidados parcializados" coordinados por un encargado, el "case manager", en la afortunada metáfora de Eisenberg (49). Los objetivos de tales "sistemas de cuidados" son mantener a los pacientes en contacto con los servicios, reducir la frecuencia y duración de las hospitalizaciones y los costos consiguientes, y mejorar los resultados, especialmente en el funcionamiento social y la calidad de vida. Es decir: el "case management" se nos propone como la solución para los problemas de seguimiento de los pacientes graves en el momento actual de pobreza, descooordinación, ineficiencia e hiperfrecuentación de los servicios de salud mental. Pero ¿cuáles son los resultados del metanálisis de los estudios aleatorizados controlados (ECA) que implicaban ese "revolucionario sistema de cuidados"? Pues parece que, al menos según el metaanàlisis de 1998, "se pierden menos pacientes" que con los sistemas "tradicionales" (sic.: ¿a qué se referirá ese término?). Pero ¡oh sorpresa!: no solo no ingresan menos en servicios hospitalarios de psiquiatría, sino que ingresan más --y, tal vez, los ingresos sean más largos. Los costes no sólo no disminuyen, sino que aumentan. Si bien hay cierta evidencia acerca de una mejoría en el cumplimiento de las prescripciones, al menos en los 
primeros tiempos del sistema, no se producen mejorías clínicas significativas en el estado mental, el funcionamiento social o la calidad de vida de los pacientes... (60). En resumen: que tal vez vaya a resultar difícil sustituir con medidas tecnoburocráticas la falta de motivación, de formación y de apoyo de los diferentes tipos de clínicos para ejercer la labor globalizadora de los cuidados del paciente, para ayudarle a integrar e integrarse -la labor que, en otras ocasiones, he llamado de "clínico referente" (para todos los avatares, recaídas, crisis y necesidades del paciente (36) (37)). Hoy se sabe que los pacientes con TMS necesitan sistemas de cuidados que tengan al menos cinco ejes fundamentales: ayuda familiar, apoyo psicológico individualizado, medios psicosociales, psicofarmacología... y sistemas de "cuidados de los cuidantes" (trabajo en equipo, grupos de reflexión y supervisión, formación continuada en servicio...). Ningún método injertado, ni aunque el esqueje provenga del Imperio -con un sistema sanitario enormemente diferente del nuestro, no lo olvidemos- va a poder sustituir la falta de formación e interés de los clínicos por esas perspectivas integradas del tratamiento.

Otro ejemplo demostrativo similar es el de alguno de los "nuevos neurolépticos" (en lo sucesivo, N.N.). Me voy a centrar en el metanaálisis de los ECA de uno de ellos, realizado dentro de las revisiones sistemáticas de la Fundación Cochrane (62). A pesar de los "miles de estudios" supuestamente realizados sobre ese NN, cuando se apura el trabajo metaanalítico, sólo se encuentran 20 estudios aleatorizados controlados valo- rables, todos ellos de cortísima duración. Resultados del metanálisis: el NN resulta más aceptable que el haloperidol; produce menos efectos secundarios $y$, desde luego, menos somnolencia... Pero todo ello en una proporción muchísimo más baja de lo que afirman las campañas de promoción de ese NN y, desde luego, con un coste muchísimo más alto que los neurolépticos de comparación. Conclusión del metanálisis: sin que se hayan realizado estudios a medio y largo plazo, hay un sesgo en la publicación absolutamente desbordante. Incluso no han sido publicados estudios con resultados negativos hoy accesibles. En definitiva, un caso más de "efecto Utah" rampante.

Pero hay quien "lo tiene peor". Aprovechando la "moda de los razonamientos analógicos sobre la fisiología cerebral basada en los neurotrasmisores" y el "efecto Utah" correspondiente (19) (27) de cualquier droga que prometa actuar sobre un neurotrasmisor diferente o mediante diferente mecanismo, se han diseñado fármacos directamente apoyados en esas perspectivas analógicas y primitivas del funcionamiento de los neurotrasmisores. Como recordaba en un trabajo teórico sobre el tema (27), después esos fármacos compiten entre sí en el sacrosanto mercado (de la salud, en este caso). Compiten en nuestros barrios, en nuestras salas de espera, en nuestras consultas y en nuestros pacientes. Compiten con una intensidad creciente, a menudo belicosa... Pero como en toda guerra, hay muertos: en el campo de batalla los muertos son reales. Algo bien obvio que demasiado a menudo parece olvidarse: ¿Cómo entender si no que, en 1998, otro de 
esos NN, que aquí prefiero no especificar, haya sido retirado del mercado, al año de haber sido introducido, tras mostrarse involucrado en varias muertes por problemas cardíacos?. ¿Qué pasó con los estudios farmacocinéticos y experimentales previos? ¿Y con los estudios clínicos pre-lanzamiento?. ¿Y con los numerosos estudios clínicos post-lanzamiento? ¿Y con el riesgo en el que se ha colocado a los mil cuatrocientos pacientes que recibieron ese mismo tratamiento 1? ¿Qué pasó con los estudios clínicos aleatorizados controlados? ¿Es que son un método falaz? $¿$ Es que los investigadores falsearon la datos? ¿Es que también los estudios aleatorizados controlados y los metaanálisis consecutivos son susceptibles de caer bajos los "sesgos del investigador" hasta el extremo de haber infravalorado tan gravemente los efectos secundarios indeseables del fármaco?... ¿Y qué pasa con las responsabilidades éticas y científicas, por no referirme aquí a las legales, de los investigadores que firmaban esos estudios?

La siguiente revisión se refiere al uso de la Terapia Electroconvulsivante (TEC), en particular con pacientes esquizofrénicos(63). Se trata de un tema sobre el cual, desde la introducción de tal terapia por Cerletti y Bini en 1936-1938, ha habido todo tipo de discusiones teóricas, técnicas y éticas. Un resumen de algunas de tales discusiones, desde una perspectiva que comparto, puede encontrarse por ejemplo en Lillo (64). Pero una revisión actualizada de los estudios acerca de tal terapia en pacientes esquizofrénicos lleva a unos resultados que les he resumido muy esquemáticamente en la tabla 5 . Es decir: a corto plazo el TEC es mejor que el TEC simulado (Pero, ¿qué tipo de actitud asistencial puede tener hacia sus pacientes un clínico para realizar un mínimo de 12 TEC simulados a un paciente? En fin: me reservo la opinión); a medio y largo plazo no hay investigaciones al respecto. A corto plazo el TEC parece mejor que el placebo... en uno de cada cinco o seis pacientes. A medio y largo plazo, es decir más allá de los 6 meses -y por supuesto, en un período científicamente razonable como serían los 5 años- no hay investigaciones al respecto valorables. La TEC, sin embargo, se muestra menos efectiva que los neurolépticos, pero no hay investigaciones a largo plazo. Es más eficaz que el coma insulínico, otro forma aún más arriesgada de "terapia de choque": pero no hay investigaciones a medio y largo plazo -y espero que no las vaya a haber. Resulta menos eficaz que la combinación de psicoterapia psicoanalítica y neurolépticos (May 1868): los únicos estudios del metaanálisis que pasan de los 2 o 3 años de duración. Es decir que hay datos empíricos que evalúan evoluciones durante al menos dos años cuyos resultados definen que la combinación de neurolépticos y psicoterapia psicoanalítica resulta más eficaz a medio y largo plazo que las tandas habituales de TEC (12 ES por tanda). Y el coste ¿sabemos cuál es el coste actual de los TEC en estos momentos, con anestesistas, enfermeros, clínicos, quirófano o similares...? Un cálculo aproximado reciente en los Servicios de Baleares (1997) era de un mínimo de 68.000 a 72.000 pts- por TEC, es decir, 840.000 pts- por tratamiento (tanda de 12). Ni siquiera se puede decir por tanto que sea un tratamiento eficiente, por lo 
que hay que pensar que se mantiene en el arsenal terapéutico ante las ansiedades asistenciales de los profesionales, el biologismo de su formación y su ignorancia o dificultad para aplicar técnicas psicológicas y psicosociales en esos servicios. En definitiva, en estos momentos parece que la TEC es un tratamiento para efectos a corto plazo, un tratamiento para dar el alta, una "terapia" para servicios sobrecargados, mal dotados de medios humanos y de técnicas psicológicas y psicosociales 2. Evidentemente, estoy exagerando la situación... pero con una base empírica para mis "propuestas" similar a la de la TEC: un sistema tal vez útil para servicios sobrecargados, mal dotados de personal y de técnicas psicololgicas y psicociales propias o de sus dispositivos de apoyo. Un sistema al cual, tal vez, pueda quedarle como indicación la del tratamiento inicial de trastornos esquizofrénicos agudos en mujeres lactantes del tercer y cuarto mundos...

Algunas.palabras sobre la droga del final del siglo: los antidepresivos, muestra sobresaliente de los logros finiseculares del "efecto Utah" y cuna de más de un "fenómeno Darsee". Para no extenderme, me limitaré a insertar aquí sendos resúmenes procedentes de un autor de clara inserción en la línea de la Psiquiatría Biológica como es J. Vallejo y de las conclusiones de un metaanálisis sobre la eficacia de los ATD en niños y adolescentes de la Universidad de Newcastle.

“...Sorprendentemente, en la extensa bibliografía actual sobre nuevos antidepresivos, sólo se recogen 16 publi- caciones de trabajos controlados en los que éstos se comparen con tricíclicos o tetracíclicos en el tratamiento de las depresiones endógenas melancólicas, de las cuales sólo tres eran metodológicamente correctos. En dos de ellas, la clomipramina resultó ser superior al citalopram y a la paroxetina, y en la tecera, al paroxetina tuvo una eficacia similar a la amitriptilina. (...) Escaso es, por tanto, el bagaje bibliográfico con el que los nuevos antidepresivos se enfrentan a los tricíclicos, y aunque se precisan más estudios que acaben de esclarecer la cuestión, todo parece indicar que estos nuevos fármacos no superan ni igualan a los clásicos en el tratamiento de la melancolía." (66)

Por otra parte, en las conclusiones del metaanálisis probablemente más amplio de los efectos de los ATD en la infancia y la adolescencia, Hazell y colaboradores(67) afirmaban taxativamente: "Los antidepresivos tricíclicos resultan no ser más efectivos que el placebo en el tratamiento de la depresión de niños y adolescentes". Es un tema con graves repercusiones, sobre todo ahora que se está proponiendo la extensión masiva de los nuevos antidepresivos al "tratamiento" de diversos trastornos psico(pato)lógicos de los niños.

Claro que los dogmas y excesos de la "perspectiva Bambi" también merecen atención y cuidado. En el primer y segundo apartados de este trabajo he intentado su crítica desde el punto de vista teórico-técnico: pongamos ahora alguna ilustración desde el punto de vista pragmático y técnico. 
Una técnica aparentemente "blanda", ecológica, casi contracultural, es la de la inmersión en agua durante el embarazo y el parto, cuyas excelencias físicas y psicológicas han sido defendidas durante largos años, incluso en ciertos medios obstétricos europeos (68). De este procedimiento, inesperadamente, existen numerosos estudios: tal vez por ese hálito "contracultural" de la técnica. Por ello ha sido posible revisar toda una serie amplísima de variables biopsicosociales que, según sus defensores, esa técnica parecía mejorar: pero el resultado del meta-análisis de Nikodem(69) es aplastante. No hay ningún evidencia significativa a favor del uso de tales sistemas, aunque tampoco se han encontrado efectos adversos para la mujer "aunque la posibilidad de resultados adversos para el neonato no debe ser ignorada". Es evidente, sin embargo, que el montaje de estos embarazos y partos es mucho más complejo y tal vez más caro 3 .

$\mathrm{Y}$ sin embargo, si hay datos a favor de técnicas que después nuestra red asistencial no utiliza... y no precisamente por su elevado coste. Me refiero, sobre todo, a algunas técnicas psicológicas y psicosociales como, por ejemplo, la del apoyo psicológico familiar sistemático en la esquizofrenia (que no es lo mismo, desde luego, que el "yo veo a la familia de vez en cuando"). De los numerosos estudios al respecto, al menos 12 cumplen los criterios para ser considerados ECA (70). Y los resultados del metaanálisis son espectacularmente claros: disminuye significativamente la frecuencia de recaídas, disminuye la hospitalización, aumenta el cumplimiento y aumenta la conservación del trabajo, aunque, a pesar de las ilusiones que algunos se habían hecho al respecto, no cambia ni la carga familiar ni las emociones soportadas por la familia... (¡Pobre familia, la del paciente esquizofrénico actual!: para cualquier clínico sensible, está bien claro lo que tiene que soportar y la escasa ayuda social al respecto). Sin embargo los costes disminuyen entre el 20 y el $58 \%$ en el grupo en el que se hacen intervenciones familiares sistematizadas de corte cognitivo o de corte psicoanalítico o sistémico. Parece por lo tanto que sería un sistema aceptable para los clínicos, para los pacientes y para sus familias, pero ¿y para los gestores?. Pues corremos el riesgo de que por el tema de los "presupuestos a corto plazo", anuales, inciertos, los gestores sigan resistiéndose porque, para evitar una recaída de un paciente por año, se necesita haber intentado hacer la intervención en 6 o 7 familias.

Por último, dada la relevancia del tema, recordemos algunas investigaciones acerca de la atención a la depresión puerperal. Como es sabido, se trata un trastorno que implica un gran sufrimiento en las familias con una madre afectada. Además, es uno de los factores de riesgo psico(pato)lógicos más reconocidos para los hijos (71-74) (37). Parece pues que cualquier esfuerzo eficaz para la ayuda en ese tema se convertiría en terapéutica y preventivamente eficiente, oportuno, y con grandes repercusiones sobre el balance autonomía / heteronomía y la calidad de vida. De ahí que sean tan importantes las investigaciones sobre los medios psicológicos y psicosociales para ayudar en el embarazo y los primeros meses de la relación madre-hijo (72). Ray y 
Hodnett(75) han realizado un meta-análisis de los estudios que implican cualquier tipo de cuidado, psicológico o psicosocial, a cargo de "cuidadores", en la depresión puerperal. Los resultados a medio y largo plazo sobre la madre y el niño aparecen reseñados en Meissels y Shonkoff (72). Los resultados sobre la depresión puerperal materna, a pesar del pequeño número de casos estudiados mediante la metodología MBE, parece que van a favor de que la depresión postparto puede ser aliviada de forma importante con medidas psicológicas y psicosociales incluso mínimas, como el "seguimiento telefónico". Probablemente, sobre la efectividad, eficacia y eficiencia de las medidas psicológicas y psicosociales en la depresión puerperal coincidiríamos casi todos los que tenemos alguna experiencia en la ayuda psicológica y psicosocial en el embarazo y las primeras relaciones padres-hijos (37) (76).

\section{Algunas reflexiones y propuestas para concluir}

En resumen: las reflexiones anteriores implican la posibilidad no sólo teórica y técnica, sino incluso práctica, de realizar todo un replanteamiento de la investigación y la docencia en los servicios y sectores (sanitarios) secundarios de los países secundarios del Imperio. En la tabla 6 aparece la sistematización de una serie de propuestas para la investigación y la docencia en salud (mental) consecuente con esas reflexiones. Las líneas directrices de ese replanteamiento de la investigación y la docencia en salud (mental) podrían ser dos: mejorar la difusión de las investigaciones y experiencias "nativas" y mejorar su eficacia, eficiencia y efectividad, es decir, su valor real para cambiar algo la existencia de todos nosotros y de nuestros consultantes, reales y potenciales. Si queremos resistirnos a la invasión por técnicas e investigaciones foráneas, poco adaptadas y eficientes en muchos casos, pero apoyadas por los medios de difusión, agencias de noticias norteamericanas y el "pensamiento único", deberíamos concentrarnos, al menos en esos sectores y ámbitos secundarios, en esa mejora de la efectividad de la investigación y la docencia y no tanto en las investigaciones realizadas para el prestigio del equipo investigador. En ese sentido, además, son tan válidas las investigaciones técnicas y tecnológicas como las científicas, y en las tres ciencias básicas de nuestro ámbito, y no solo en biología. Habría que reinvindicar el valor de las metodologías no empiristas, que sí utilizan los sociólogos, así como el valor científico de algunos métodos observacionales... Habría que reivindicar orientaciones de la investigación menos unidimensionales: investigar las técnicas y tecnologías no sólo en su eficacia, sino también en su eficiencia, efectividad, accesibilidad, oportunidad, etc (Tabla 3).

Necesitamos una investigación y una docencia más contextualizada con el estado, intereses y orientaciones de nuestras redes de salud mental concretas, intentando siempre integrar los diversos escalones de la asistencia y la docencia; no siguiendo, como siempre, el modelo en el que los hospitales de alta tecnología y los centros universitarios son los "altares del saber" ... con el resultado de que, a menudo, ese saber resulta muy poco válido para la asistencia y para los clínicos que la realizan o, para cuando es eficaz, 
se ha hecho un grave dispendio durante años o decenios, como en el caso de la investigación en psicoterapia en las últimas décadas. La integración creo que ha de llegar a la la integración de Investigación y Desarrollo (I+D) e Investigación y Empresa (I+E) ya que, nos guste no o nos guste, vivimos en un país que ha votado una Constitución como "economía social de mercado" (aunque el elemento social aparezca cada día más desleído o desvalorizado). Resulta por tanto perfectamente legal y hasta honesto que las empresas investiguen y realicen sus investigaciones y la promoción de sus productos: las organizaciones profesionales, la administración y las organizaciones empresariales deberíamos pactar sistemas de colaboración y códigos éticos mínimos para esa colaboración, más que seguir abonando la situacion actual, en la cual el descontento puede oírse en los tres sectores, pero ante temores corporativos y de represalias comerciales, nadie se atreve a "ponerle el cascabel al gato"... El resultado es bien conocido: derroche de medios para investigación, docencia y promocióón de productos, corruptelas variadas, efectos Utah repetitivos y ensordecedores, encarecimientos y sesgos intolerables en la formación y la docencia, violencia en las relaciones entre los clínicos y los vendedores... Por el contrario ¿no sería posible que determinadas empresas, Institutos o Fundaciones proporcionaran los medios para integrar los hasta hoy dispersos intentos de investigación y potenciar los medios administrativos de las pequeñas instituciones con intereses investigadores (como los Centros de Salud Mental)? Se podría potenciar la realización y difusión de sus investigaciones, apoyar la docencia adaptada a las necesidades biopsicosociales de la red, y no sólo actividades de promoción mercantiles, en función de intereses estrechamente comerciales y a corto plazo... Sería la única forma de que esa necesaria dedicación a "buscar financiación" (que se lleva hasta el $85 \%$ del tiempo de los equipos de investigación, al menos en los países y sectores secundarios del Imperio) no de al traste con los intereses de investigación de muchas instituciones "blandas", comunitarias. Acuerdos con la industria, "fundación de fundaciones", institutos bibliográficos y de gestión, Institutos para la Educación Sanitaria (como el IES catalán), etc podrían retroalimentar nuestras investigaciones, considerando, como antes decía, otras variables a investigar, y realizando investigaciones clínicoasistenciales más realistas --que implican, desde luego, metodologías y presupuestos a medio plazo ( 2 a 5 años) y planteamientos integradores biopsicosociales.

En resumen, se trata de perspectivas de la investigación y la docencia tal vez menos apoyadas en las supuestas "ciencias básicas", tal vez menos grandiosas en sus planteamientos, con resultados menos espectaculares... Pero propongo pensar si esos "maravillosos descubrimientos" que nos anuncian cada día, esos anuncios fulgurantes de "bálsamos y técnicas de Fierabrás", esos anuncios de un azul y a-problemático futuro asistencial gracias al desarrollo de las técnicas biológicas y mecánicas, ¿esos anuncios son o van a suponer realidades? ¿Proponen realidades o son tan solo una ficción, una forma de consolidar el "efecto Utah", una forma de negar y disociar algo tan básico como las 
dificultades y la complejidad de nuestro trabajo asistencial? (tabla 7). Por el contrio, algunos pensamos que nuestro trabajo es y va a seguir siendo complejo, problemático, con infrecuentes resultados de "curación total" y que no poseemos ninguna panacea, salvo la de trabajar lo más y mejor posible en prevención.

Nuestro trabajo en el campo de la asistencia psico(pato)lógica y de salud mental hay que reconocer que hoy en día resulta a menudo poco eficaz y eficiente, al menos para los ideales de la medicina. Cuando un cuadro psicopatológico grave está instaurado, su atención implica una gran inversión de esfuerzos que, aplicada durante años y años, a miles de pacientes, con migrados resultados, deviene en extremo cronificante... incluso para nosotros mismos. De ahí esos anuncios de descubrimientos terapéuticos y sistemas docentes que "curan rápidamente" o mediante técnicas que se aprenden a manejar "rápidamente y con sencillez". De ahí que a buena parte de las perspectivas más sectarias de la investigación y la docencia (tanto en su extremo "Godzilla" como en su extremo "Bambi"), se les podría aplicar la agnóstica visión que ya hace muchos años, en nuestro hoy casi olvidado "Siglo de Oro", pergeñó Lupercio Leonardo de Argensola 4:

\section{...Porque ese cielo azul que todos vemos ni es cielo, ni es azul. \\ ¡Lástima grande que no sea verdad tanta belleza!}

\section{Lupercio Leonardo de Argensola} (circa 1.600)

\section{NOTAS}

\footnotetext{
${ }^{1}$ Como señalé en ese otro trabajo, resulta una impresionante muestra de la guerra comercial a la que me refiero y del uso del efecto Utah, lo que la propia empresa fabricante, en la monografía de lanzamiento, atestigua: En su apartado de "seguridad y tolerabilidad" se afirma que "un total de 1.436 pacientes han sido tratados con el producto en estudios a largo plazo; de ellos, 400 han seguido tratamiento durante más de un año". Llama la atención que baste tratar a 1.436 pacientes para lanzar un producto que puede ser prescrito como poco al $0,2-0,5 \%$ de la población y que se considere un "estudio de seguridad y tolerabilidad a largo plazo" uno que, como media, ¡¡no dura ni un año!!
}

\footnotetext{
2 Porque, si de los que se trata es de dar altas rápidas, sin respetar ni tan siquiera la integridad mental de los pacientes (May 1968, Lillo 1997), ¿por qué no cerrar las puertas de los servicios o empeorar aún más la calidad de la vida en los mismos? Se lograría también evitar ingresos, dar más altas y hacer las estancias más breves...

${ }^{3}$ Y en nuestro caso si tenemos alguna evidencia (clínica, claro está) procedente de neonatos con infecciones después de alguno de estos procedimientos.

${ }^{4}$ Por cierto que sobre unos conocimientos físicos y una perspectiva epistemológica bastante avanzada, incluso para nuestros días.
} 
Tabla 1. EL PROBLEMA DE LOS TRATAMIENTOS EXCLUSIVAMENTE FARMACOLÓGICOS DE LOS TRASTORNOS MENTALES MÁS PREVALENTES EN A.P.S.M.

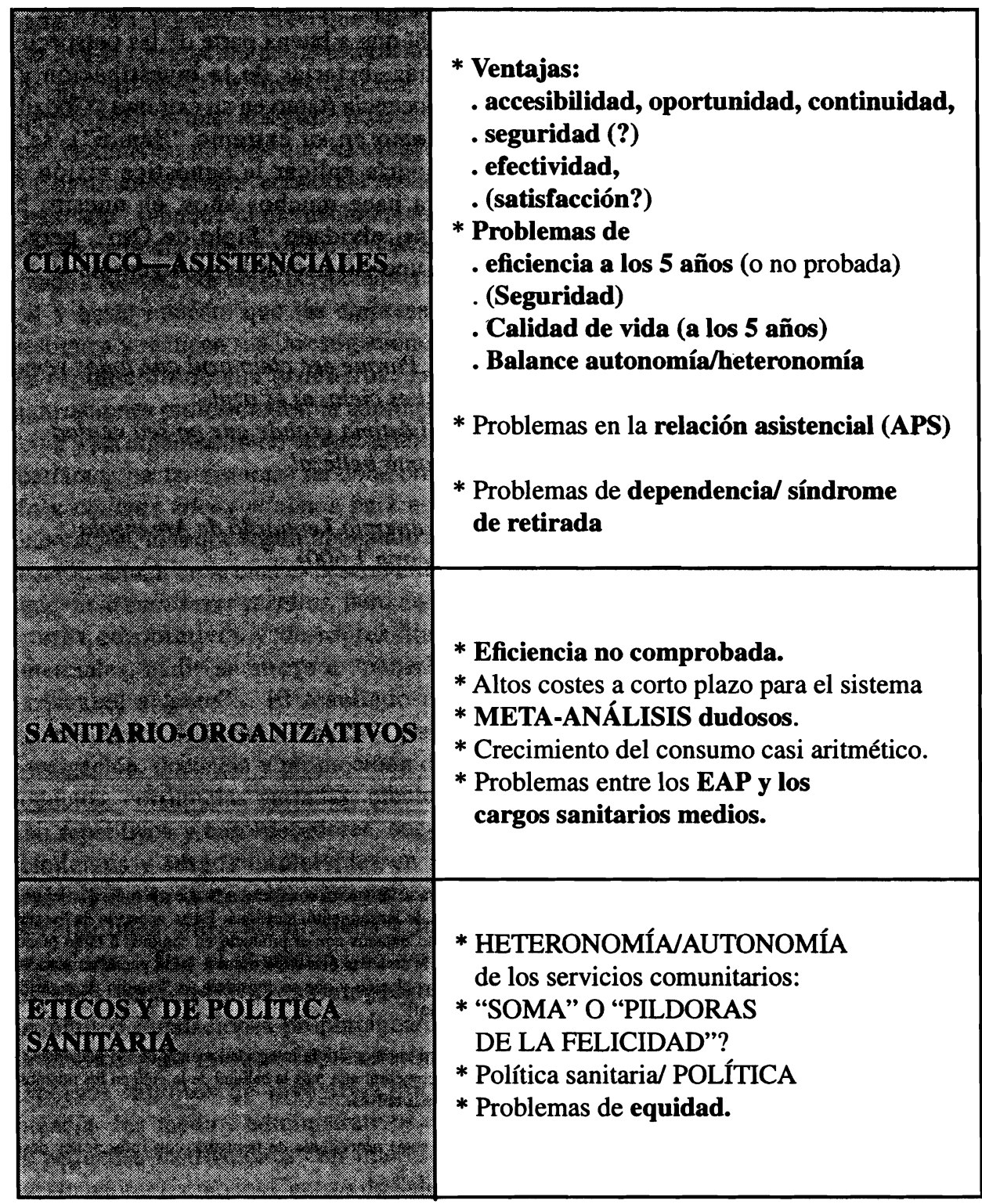

(Derivada de los datos de Kazdin 1990, Ruiz Doblado 1998, Vallejo 1997, Anderson 1995...) 
DEBATES

Tabla 2. AREAS QUE DEBERÍAN PRIORIZARSE EN LA INVESTIGACIÓN DE LAS PSICOTERAPIAS

$$
\text { (derivada de Kazdin }(1990,1991)
$$

\section{1) MUESTRAS CLÍNICAS}

(habitualmente infrarrepresentadas con respecto a las muestras recogidas en encuadres "no clínicos": análogos, voluntarios, universitarios, etc.);

2) Investigación de los EFECTOS ESPECÍFICOS sobre problemas, cuadros, personalidades, poblaciones u otras CARACTERÍSTICAS ESPECÍFICAS.

3) Investigaciones en casos de "co-morbilidad" (CO-DIAGNÓSTICO), con especial hincapié en los efectos diferenciales de cada técnica y sistema (con respecto a cada uno de esos problemas)

4) Focalizar más las investigaciones hacia POBLACIONES

. infradotadas de medios psicosociales

. poblaciones de riesgo.

5) Atención especial a las TÉCNICAS INFRAESTUDIADAS, pero frecuentemente utilizadas,

p.ej., los tratamientos de "marco de referencia" psicoanalítico.

6) Investigación de los resultados de las TÉCNICAS ADAPTADAS:

. combinación de tratamientos,

. simplificación de tratamientos

. sistemas breves.

7) Investigar no sólo la eficacia o la eficacia comparada, sino las ONCE CARACTERÍSTICAS DE LA CALIDAD DE CUALQUIER TERAPIA resumidas en la tabla adjunta. 


\section{Tabla 3. COMPONENTES DE LA CALIDAD DE UNA TERAPIA CUALQUIERA}

\begin{tabular}{|c|c|}
\hline 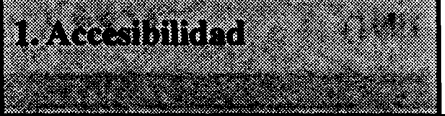 & $\begin{array}{l}\text { = Facilidad con la cual los consultantes pueden } \\
\text { obtener atención cuando la necesitan. }\end{array}$ \\
\hline 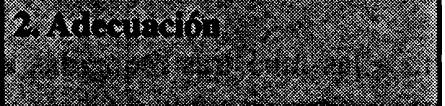 & $\begin{array}{l}=\text { Forma de provisióón de un cuidado o terapia, } \\
\text { según el estado del desarrollo tecnológico. }\end{array}$ \\
\hline & $\begin{array}{l}=\text { La atención necesaria está coordinada entre } \\
\text { diferentes profesionales, dispositivos, organizaciones } \\
\text { y a lo largo del tiempo. }\end{array}$ \\
\hline 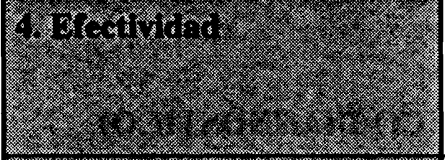 & $\begin{array}{l}\text { = Las técnicas o procedimientos se proporcionan } \\
\text { de forma adecuada, según el desarrollo tecnológico } \\
\text { medio ("eficacia real"). }\end{array}$ \\
\hline & $\begin{array}{l}\text { = Capacidad de una técnica para solucionar los } \\
\text { problemas para los cuales está indicada. }\end{array}$ \\
\hline & $\begin{array}{l}=\text { Consumo de esfuerzos, economía, personal, } \\
\text { repercusiones indeseables, etc de una técnica. }\end{array}$ \\
\hline & $\begin{array}{l}=\text { Grado en el cual los procedimientos están libres de } \\
\text { azar o peligros potenciales. }\end{array}$ \\
\hline 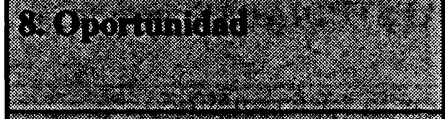 & $\begin{array}{l}\text { = Grado en el cual la atención se proporciona cuando } \\
\text { se necesita. }\end{array}$ \\
\hline 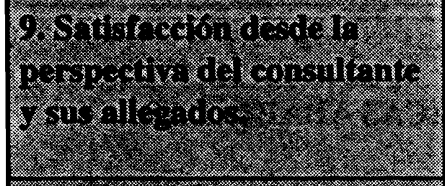 & $\begin{array}{l}=\text { Evaluación "objetiva" y mediante entrevista de la } \\
\text { satisfacción de los mismos. }\end{array}$ \\
\hline mingor & $\begin{array}{l}\text { = Variación en la calidad de vida, evaluada según los } \\
\text { sistemas habituales, entre el "antes" y el "después" } \\
\text { (o "durante") de una terapia. }\end{array}$ \\
\hline 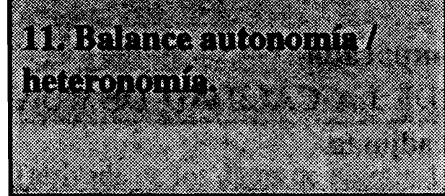 & $\begin{array}{l}\text { = Grado de incorporación de los pacientes y sus } \\
\text { familias en los procesos de decisión de la técnica y en } \\
\text { los procesos y procedimientos exploratorios } \\
\text { y terapéuticos. }\end{array}$ \\
\hline
\end{tabular}


Tabla 4. CONDICIONES ACTUALES PARA UNA INVESTIGACIÓN CIENTÍFICA O TECNOLÓGICA EFICIENTE Y ADECUADA EN SALUD MENTAL (derivada de Garske y Lynn, 1988)

. Grupos homogéneos de consultantes con problemas específicos e idénticos

. Muestra suficientemente numerosa

. Asignados aleatoriamente a las condiciones experimentales

- Terapeutas igualados en cuanto a variables críticas

- Grupos de control cuidadosamente diseñados o bien DISEÑOS DE CASO ÚNICO

-Y que sean administradas en varias oportunidades:

Duración mínima de un estudio en salud mental: de 2 a 5 años.

. Muestra y procedimientos bien descritos

. Con tribunales de estilo.

. Considerando la pragmática de la investigación:

. Para qué se publica?.

. "Colegios invisibles"

- * En último extremo lo que recibimos lo aceptamos por CIENCIA o por CONFIANZA Y FE? 
Tabla 5. RESULTADOS DE LA TERAPIA ELECTROCONVULSIVANTE EN LA ESQUIZOFRENIA.

\begin{tabular}{|c|c|}
\hline 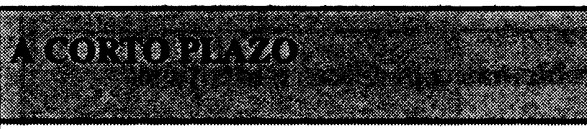 & 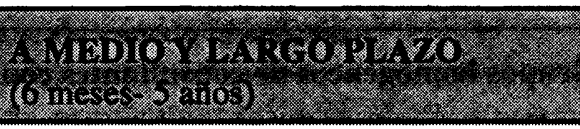 \\
\hline . Mejor que el "TEC simulado" (?) & . sin investigaciones \\
\hline . Mejor que el placebo & . sin investigaciones \\
\hline Menos eficaz que los NLT & . sin investigaciones \\
\hline $\begin{array}{l}\text { La combinación de TEC y NLT mejora } \\
\text { los resultados. }\end{array}$ & . evidencias equívocas \\
\hline . Más eficaz que el coma insulínico (?) &.$?$ \\
\hline $\begin{array}{l}\text { Menos eficaz que la combinación de } \\
\text { Psicoterapia Psicoanalítica y NLT } \\
\text { (May 1968) }\end{array}$ & $\begin{array}{l}\text {. Es el único estudio que siguió más allá } \\
\text { de los dos años. }\end{array}$ \\
\hline $\begin{array}{l}\text { Coste de un tratamiento estimado } \\
\text { en pesetas de } 1998 \text { : } \\
70.000 \text { pts } \times 12 \text { sesiones }=840.000 \text { pts }\end{array}$ &.$? ? ?$ \\
\hline \multicolumn{2}{|c|}{$\begin{array}{l}\text { Es decir: Se trata de una técnica: } \\
\text {. sòlo estudiada a corto plazo } \\
\text {. orientada a "altas rápidas" } \\
\text {. casi sin base empírica o teórica que la apoye }\end{array}$} \\
\hline $\begin{array}{l}\text { Utilizada por lo tanto en servicios: } \\
\text {. sobrecargados } \\
\text {. mal dotados } \\
\text {. que no poseen o no saben utilizar }\end{array}$ & nicas psicológicas y psicosociales \\
\hline
\end{tabular}


DEBATES

Tabla 6. PROPUESTAS PARA LA INVESTIGACIÓN Y LA DOCENCIA EN SALUD (MENTAL) (I)

\begin{tabular}{|c|c|}
\hline 1* Tanto científica & * como tecnológica y técnica \\
\hline $\begin{array}{l}\text { 2* En las TRES CIENCIAS BÁSICAS } \\
\text {. Inv. Psicológica y psicosocial } \\
\text {. Inv. Biológica } \\
\text {. Inv. Sociológica }\end{array}$ & $\begin{array}{l}\text { * Sin desdeñar los temas de } \\
\text { PRAGMÁTICA ASISTENCIAL }\end{array}$ \\
\hline $\begin{array}{l}\text { 3* Revalorizando METODOLOGÍAS } \\
\text { NO EMPIRISTAS }\end{array}$ & $\begin{array}{l}\text { * Que, a menudo, sí usan: } \\
\text {. los sociólogos } \\
\text { * Que, a menudo, sí usan: } \\
\text {. y los especialistas en metodología } \\
\text { observacional. }\end{array}$ \\
\hline $\begin{array}{l}\text { 4* Menos UNIDIMENSIONALES } \\
\text { en las características a investigar } \\
\text { o difundir }\end{array}$ & $\begin{array}{l}\text { * de la EFICACIA } \\
\text { * pero también de la EFICIENCIA, } \\
\text { ACCESIBILIDAD, OPORTUNIDAD, } \\
\text { CALIDAD DE VIDA, } \\
\text { AUTONOMÍA/HETERONOMÍA... }\end{array}$ \\
\hline 5* Más CONTEXTUALIZADAS & $\begin{array}{l}\text { * Según las características y necesidades } \\
\text { del país } \\
\text { * En el encuadre habitual de los ESM } \\
\text { (poblacional, territorializado...) }\end{array}$ \\
\hline
\end{tabular}


Tabla 6. (cont). PROPUESTAS PARA LA INVESTIGACIÓN Y LA DOCENCIA EN SALUD (MENTAL) (continuación)

\begin{tabular}{|c|c|}
\hline $\begin{array}{l}\text { 6* INTEGRANDO, con líneas } \\
\text { contextualizadas }\end{array}$ & $\begin{array}{l}\text { * Hospital - Universidad } \\
\text { * Hospital - ESM - Otros dispositivos } \\
\text { * ESM- Universidad }\end{array}$ \\
\hline 7* INTEGRANDO I + D // I + E & $\begin{array}{l}\text { - Empresas químico-farmacéuticas } \\
\text { - Empresas concertadas } \\
\text { - I+D no es "Investigación + Destrucción" } \\
\text { (investigación en programas bélicos) }\end{array}$ \\
\hline $\begin{array}{l}\text { 8* INTEGRANDO LOS } \\
\text { EQUIPOS DE APOYO }\end{array}$ & $\begin{array}{l}\text { * Para la búsqueda de Financiación. } \\
\text { * Para la difusión de las investigaciones } \\
\text { realizadas en nuestro ámbito. } \\
\text { * Para la publicación. }\end{array}$ \\
\hline & $\begin{array}{l}\text { FUNDACIÓN DE FUNDACIONES } \\
\text { INSTITUTOS BIBLIOGRÁFICOS }\end{array}$ \\
\hline $\begin{array}{l}\text { 9* Considerando la EFICIENCIA } \\
\text { científica y tecnológica de las } \\
\text { INVESTIGACIONES CLfNICAS } \\
\text { con SEGUIMIENTO adecuado. }\end{array}$ & * 2 a 5 años y longitudinales \\
\hline $\begin{array}{l}\text { 10* INVESTIGACIONES } \\
\text { CLINICO-ASISTENCIALES } \\
\text { (pragmáticas, tecnológicas...) }\end{array}$ & $\begin{array}{l}\text { codiagnóstico } \\
\text {. combinación de tratamientos } \\
\text {. seguimientos y abandonos } \\
\text {. continuidad de cuidados (_hoy?) } \\
\text { programas preventivos (P.e. primera infancia) } \\
\text {. técnicas “adaptadas”... }\end{array}$ \\
\hline
\end{tabular}


DEBATES

Tabla 7. LA APROXIMACIÓN CLÍNICA EN EL SIGLO XXI

(¿Más allá de GODZILLA y BAMBI?)

\begin{tabular}{|c|c|}
\hline 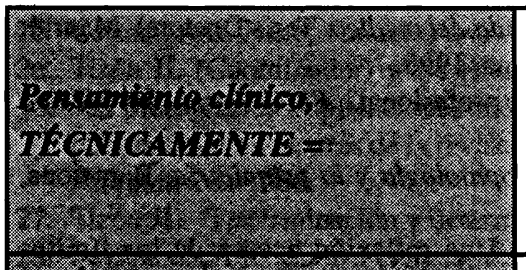 & $\begin{array}{l}\text { * Junto al paciente } \\
\text { * Respetando su autonomía } \\
\text { * Globalizando } \\
\text { * No ruralista/elitista }\end{array}$ \\
\hline 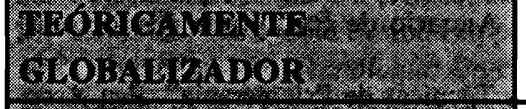 & * Pero con globalización operativizada \\
\hline 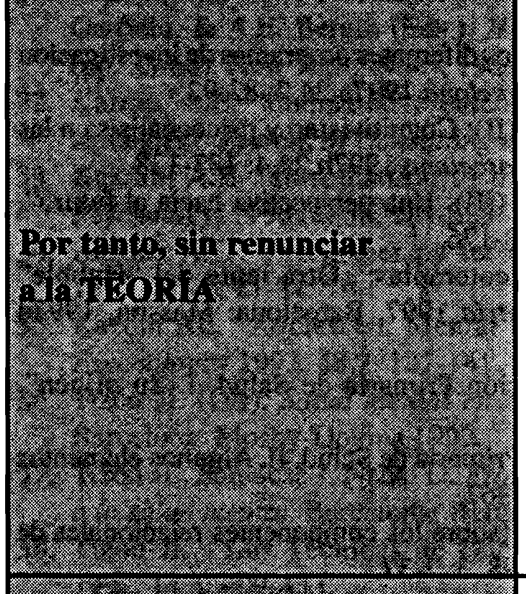 & $\begin{array}{l}\text { * No reduccionista, pero si especializado. } \\
\text { * Pensamiento de Equipo } \\
\text { o Grupo/ no narcisismo asistencial. } \\
\text { * Tecnologías blandas (PS) } \\
\text { * Placer/trabajo creativo en la } \\
\text { definición de salud. } \\
\text { * Abierta a los nuevos fenómenos sociales: } \\
\text {. familiares } \\
\text {. de individuación progresiva } \\
\text {. la importancia de los "media" } \\
\text {. la crítica de la extrodeterminación... }\end{array}$ \\
\hline 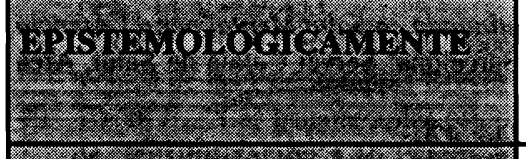 & $\begin{array}{l}\text { * Pluralismo metodológico } \\
\text { * Constructivismo y construccionismo. }\end{array}$ \\
\hline 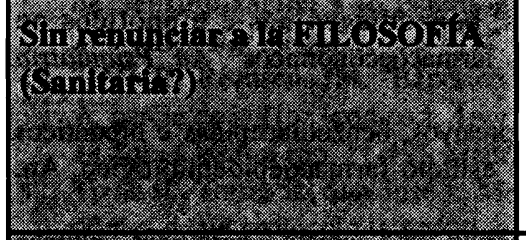 & $\begin{array}{l}\text { *Atención Sanitaria Centrada en el } \\
\text { Consultante también en S.M. } \\
\text { * Centrípeta con respecto a los Sistemas } \\
\text { Profanos y a la Red Social. }\end{array}$ \\
\hline 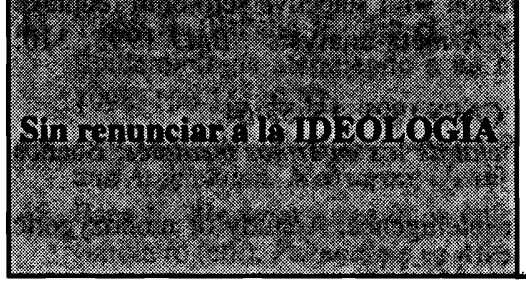 & $\begin{array}{l}\text { *Autonomizante } \\
\text { * Comunitarismo / Equitatividad } \\
\text { * No al productivismo y al Sobrevivencialismo. } \\
\text { * Democracia es (también) respeto y } \\
\text { valoración del pensamiento minoritario. }\end{array}$ \\
\hline
\end{tabular}




\section{BIBLIOGRAFIA}

1. Bell D. "Primitive Mind of State". Psychoanalytic Psychotherapy 1997, 10,1: 45-57.

2. Mingote JC. Satisfacción, estrés laboral y calidad de vida del médico. Tesis Doctoral. Madrid: Universidad Autónoma de Madrid, Facultad de Medicina, 1995.

3. Mingote JC. "Síndrome burnout o síndrome de desgaste profesional". FMC-Formación Médica Continuada en Atención Primaria 1998,5,8: 493-503.

4. Tizón JL. Introducción a la epistemología de la psicopatología y la psiquiatría. Barcelona, Ariel, 1978.

5. Tizón JL. ¿Existen las "ciencias interpretativas”?: Una reflexión acerca de los límites epistemológicos del conocimiento psicoanalitico. Anuario de Psicología (Barcelona) 1995, 67: 51-75.

6. Tizón JL. "Una propuesta de conceptualización de las Técnicas de Psicoterapia". Rev. Asoc. Española de Neuropsiquiatría 1992, 43: 283-295.

7. Tizón JL. "Cognitivismo, psicoanálisis, psicobiología (I): ¿diferentes programas de investigación para un mismo objeto?" Rev. Psiquiatría Fac.Med. Barcelona 1997a,24,2: 82-92.

8. Tizón JL. "Cognitivismo, psicoanálisis, psicobiología (II): Cognitivismo y psicoanálisis en las técnicas de psicoterapia”. Rev. Psiquiatría Fac.Med. Barcelona 1997b,24,4: 133-138.

9. Tizón JL. "Cognitivismo, psicoanálisis, psicobiología (III): Una perspectiva hacia el futuro". Rev. Psiquiatría Fac.Med. Barcelona 1997c, 24, 6: 163-176.

10. Tizón JL. "La investigación empírica sobre las psicoterapias: ¿Otra lente del "Hubble" defectuosa?" En J. Vallejo (dir.). Update de Psiquiatría 1997. Barcelona: Masson, 1997d (pp. 67-99).

11. Tizón, JL. "Los "Grupos de Reflexión" en la Atención Primaria de Salud. I. Su origen". Atención Primaria 1993,6: 309-314.

12. Tizón, JL. "Los "Grupos de Reflexión" en la Atención Primaria de Salud. II. Algunos elementos teóricos y técnicos". Atención Primaria. 1993, 7: 361-367.

13. Tizón JL. ¿"Grupos Balint" o "Grupos de Relfexión" (sobre los componentes relacionales de la asistencia?" Temas de Psicoanálisis (Barcelona) 1998, 3: 7-37.

14. Tizón JL (coord). Grup de Treball sobre Formació Continuada en Salut Mental per a l'Atenció Primària de Salut. Informe Final i Recomenacions. Barcelona: Servei Catalá de Salut, Area Sanitária, Generalitat de Catalunya 1997.

15. Pool R. “Utah Effects Strikes Again?" Science 1989, 244: 420.

16. Ruiz S, Pérez A. "Utilización de antidepresivos, benzodiacepinas e hipnóticos no benzodiacepínicos en atención primaria (I): Aspectos farmacoeconómicos". An. Psiquiatría (Madrid) 1997:13,10: 426-431.

17. Ruiz S, Torrecilla A, Pérez A. Utilización de antidepresivos, benzodiacepinas e hipnóticos no benzodiacepínicos en atención primaria (II): un estudio farmacoepidemiológico. An. Psiquiatría (Madrid) 1998:114,1: 1-7.

18. Anderson IM, BM Tomenson. "Treatment discontinuation with selective serotonin reuptake inhibitors compared with tricyclic andidepressants: A meta-analysis". BMJ 1995, 310: 1433-1438.

19. Clavero J. "La investigación bajo sospecha". Quadern CAPS 1991, 15: 55-64.

20. Goofman E. Internados: Ensayo sobre la situación social de los enfermos mentales. Buenos Aires: Amorrortu 1972.

21. Burt C. "The genetic determination of differences in intelligence: A study of monozygotic twins reared together and apart". B. J. Psychol. 1966, 57: 137-153. 
DEBATES

22. Eysenck EH. Raza, inteligencia y educación. Barcelona: Aura 1973.

23. Liugman CG. El mito de la inteligencia. Barcelona: Martínez Roca, 1972.

24. Kamin LJ. The Science and Politics of IQ. Hamondsworth: Penguin 1974.

25. Colman AM. Facts, Fallacies and Frauds in Psychology. London: Unwin Hyman 1987.

26. Tizón JL (Coordinador) y De la Lama E, Díaz-Munguira JM, Salamero M (Compiladores). "El biologismo: implicaciones teóricas, repercusiones en la asistencia sanitaria". (2 tomos publicados como extras de la revista "Informaciones Psiquiátricas"). Barcelona: Informaciones Psiquiátricas 1986.

27. Tizón JL: "Determinación y determinismo de la Psicopatología". 1999: (en preparación).

28. Feyerabend PK. Contra el método. Barcelona: Ariel 1974.

29. Editorial. "How Cold Fusion Happened - Twice”. Science 1989, 244: 420.

30. Quintanilla, MA. Tecnología: Un enfoqe filosófico. Madrid: Fundesco 1989.

31. Kazdin, A.E. "The evaluation of psychotherapy: Research design and methodology". En S.L. Garfield, \& A.E. Bergin (Eds.), Handbook of psychotherapy and behavior change (3rd ed.) (pp. 23-68). New York: Wiley, 1986 (a).

32. Kazdin, A.E. "Comparative outcome studies of psychotherapy: Methodological issues and strategies". Journal of Consulting and Clinical Psychology, 1986 (b) 54, 95-105.

33. Kazdin, A.E. "Introduction to the Special Series". Journal of Consulting and Clinical Psychology, 1990 (a),58, 681-683.

34. Kazdin, A.E. "Psychotherapy for children and adolescents". Annual Review of Psychology, 1990 (b),41, 21-54.

35. Kazdin AE. "Adolescent Mental Health: Prevention and Treatment Programs". American Psychologist 1993, 48,2: 127-141.

36. Tizón JL (Dir). Salud mental en Atención Primaria y Atención Primaria en Salud Mental. Barcelona: Mosby-Doyma 1992.

37. Tizón JL, San-José J, Nadal D. Protocolos y programas elementales para la atención primaria a la salud mental. Barcelona: Herder, 1997.

38. Tizón JL. "Las Técnicas de Psicoterapia ante la Etica”. Rev. Clínica y Análisis Grupal 1992, 14,3: 409-433.

39. Tizón JL. "Las psicoterapias psicoanalíticas breves y las "terapias de flash": I. La evolución técnica de las Psicoterapias Breves”. Informaciones Psiquiátricas 1994,138,4: 358-368.

40. Tizón, JL. "Las psicoterapias psicoanalíticas breves y las "terapias de flash": II. Los "Procesos de Sensibilización a lo Psicológico", Terapias “de Flash” o Psicoterapias ultrabreves”. Informaciones Psiquiátricas 1994,138,4: 369-392.

41. Tizón JL, Recasens JM. "Experiencias grupales en Atención Primaria de Salud”. En A. Avila y A. garcía de la $\mathrm{Hoz}$ (comps.). Aportaciones de la Psicoterapia de Grupo a la atención pública en Salud Mental (pp. 11-49). Madrid: Quipú Ediciones, 1994.

42. Tizón JL, Torres E., San-José J., Medina C, Sáinz F. "Sobre la enuresis funcional: investigaciớn clinica de un protocolo terapeutico". An.Esp. Pediatría 1995;43: 313-322.

43. Tizón JL, Pañella H. "En el centenario de los "estudios sobre la Histeria": Un servicio de Epidemiología atendiendo a un brote de Histeria Colectiva". Informaciones Psiquiátricas 1998, 150-151: 9-37.

44. Joint Commission on Accreditation of Healthcare Organizations. Primer on Indicator Development and Application: Measuring Quality in Health Care. Illinois: Joint Commission 1990.

45. Baca E. "Repercusiones de la enfermedad mental sobre la calidad de vida". En JL VázquezBarquero (ed.). Psiquiatría en Atención Primaria. Madrid: Aula Médica 1998 (pp.849-867). 
46. Lynn, R.J., y Garske, P. Psicoterapias contemporáneas. Bilbao: DDB, 1988

47. Mahoney, M.J. Human change processes. New York: Basic Books, 1991.

48. Berger R. Nazi Science: "The Dachau Hypothermia Experiments". N. Eng. J.Med. 1990, 322: 1435.

49. Eisenberg L. "The Social Construction of Human Brain". Am. J. Psychiatry 1995, 152,11: 1563- 1575.

50. Grand S, Pardes H. "The transition from Sleep to Wakefulness: Implications of a Study of the Organization of Laboratory Dream Reports for Psychoanalytic Situation". J. Amer Psychoanal. Asoc. 1974,22,1: 58-83.

51. Kinsey A, Pomeroy WB, Martin CE. Sexual behavior in the Human Male. Filadelfia: Saunders 1968.

52. Kinsey A, Pomeroy WB, Martin CE, Gebhard PH. Sexual behavior in the Human Female. Filadelfia: Saunders 1968.

53. Zecchini 1. "La otra cara de Alfred Kinsey”. El País, 1998, 22 de marzo: 17.

54. Jones JH. Alfred Kinsey: a public/private life. New York: WW Norton, 1998.

55. Burt C. "The genetic determination of differences in intelligence: A study of monozygotic twins reared together and apart”. B.J. Psychol. 1966,57: 137-153.

56. Bonfill X. "Editorial: La colaboración Cochrane". Atención Primaria 1996,18,6: 273-278.

57. Gómez A. "La medicina basada en la evidencia: aspectos controvertidos". Formación Médica Continuada en Atención Primaria 1998,5,3: 185-190.

58. Sackett D, Richardson WS, Rosenberg W, Haynes RB. Evidence based Medicina. How to practice and teach EBM. London: Churchill Livingstone 1997.

59. Tizón JL. "Los nuevos modelos asistenciales en Medicina: Una visión a partir de algunos de sus fundamentos psicológicos y epistemológicos". Revisiones en Salud Pública 1995,4: 57-83.

60. Marshall M, Gray A, Lockwood A, Green R. "Case managemente for people with severe mental disorders". En Adams CE, Duggan L, Wahlbeck K, White P (eds). Schizophrenia Module of the Cochrane Database of Systematic Reviews. Oxford: Update Software 1998.

61. Tizón JL, Bermejo J, Garre A. "Una investigación a propósito de dos años de experiencia en consultas de Neuropsiquiatría de Zona del INSALUD: II. Psiquiatría psicológicamente orientada versus Neuropsiquiatría biológicamente orientada". Informaciones Psiquiátricas 1981, 84 (3): 255-273.

62. Kennedy F, Sonf F, Hunter R, Gilbody S. "Risperidone versus "conventional" antipsychotic medication for schizophrenia". En Adams CE, Duggan L, Wahlbeck K, White P (eds). Schizophrenia Module of the Cochrane Database of Systematic Reviews. Oxford: Update Software 1998.

63. Tharyan P. Electroconvulsive therapy for schizophrenia.En Adams CE, Duggan L, Wahlbeck K, White P (eds). Schizophrenia Module of the Cochrane Database of Systematic Reviews. Oxford: Update Software 1998.

64. Lillo JL. "Apuntes para una reflexión ética sobre la terapéutica electroconvulsiva". Inform. Psiquiátricas (Barcelona). 1998, 63-66.

65. May PR. Treatment of schizophrenia. New York: Science House 1968.

66. Vallejo J. Editorial: El futuro del tratamiento de la depresión y el efecto rombo. "Psiquiatría biológica". 1998, 5,1: 1-2.

67. Hazell P, O'Connell D, Heathcote A, Robertson J, Henry D. "Efficacy of tryciclic drugs in treating child and adolescent depression: a meta-analysis". BMJ. 1995, 310: 897-900.

68. Schorn Mn, MCAllister JL, Blanco JD. "Water inmersion and the effecto on labor". $J$ Nurse-Midwifery 1993, 38,6: 336-342. 
DEBATES

69. Nikodem VC. "Inmersion in water during pregnancy, labour and birth". En Neilson JP, Crowther CA, Hodnett ED, Hofmeyr GJ (eds.). Pregnancy and Childbirth Module of the Cochrane Database of Systematic Reviews. Oxford: Update Software 1998.

70. Mari JJ, Streiner D. "Family intervention for Schizophrenia". En Adams CE, Duggan L, Wahlbeck K, White P (eds). Schizophrenia Module of the Cochrane Database of Systematic Reviews. Oxford: Update Software 1998.

71. Amigó E. et al. Medicina preventiva en l'edat Pediàtrica: Protocol de Salut Mental. Barcelona: Direcció General de SAlut Pública - Institut d'Estudis de la Salut . Barcelona: Departament de Sanitat i Seguretat Social de la Generalitat de Catalunya 1999.

72. Meissels SJ y Shonkoff JP (comps). Handbook of Early Childhood Intervention. Cambridge: Press Syndic of the University of Cambridge, 1990.

73. Lamour M, Lebovici S. "Les interactions du nourrison avec ses partenaires". Psychiatrie de l'Enfant 1991; 34,1:171-275.

74. Rutter M. De los hallazgos en la investigación a la práctica clínica. Córdoba: Fundación Castilla del Pino 1997.

75. Ray KL, Hodnett ED. "Caregiver support for postpartum depression”, En Neilson JP, Crowther CA, Hodnett ED, Hofmeyr GJ (eds.). Pregnancy and Childbirth Module of the Cochrane Database of Systematic Reviews. Oxford: Update Software 1998.

76. Tizón JL. Prevención de los problemas de salud mental del embarazo en la adolescencia. En PAPPS y SEMFYC: Curso a distancia de prevención en atención primaria. Módulos 5,6 y 7. Barcelona: SEMFyC, 1997 (pp. 59-75).

*Psiquiatra de Atención Primaria y Psicoanalista.

Director de la Unidad de Salud Mental "Sant Martí-Nord"

Institut Catalá de la Salut. Barcelona.

Dirección: Unitat de Salud Mental de Sant Martí-Nord (I.C.S.)

Plaza de la Infancia, s/n - 08020 - Barcelona.

Fecha recepción: 13-8-00 